\title{
DECISIONS BASED ON A GENERAL PREMIUM PRINCIPLE IN CUMULATIVE PROSPECT THEORY
}

\author{
Endre Pap ${ }^{1}$, \\ Ana Simićević1, \\ Biljana Mihailović2, \\ Mirjana Štrboja ${ }^{3}$
}

\author{
${ }^{1}$ Singidunum University, \\ Belgrade, Serbia \\ ¿University of Novi Sad, \\ Faculty of Technical Sciences, \\ Novi Sad, Serbia \\ ${ }^{3}$ University of Novi Sad, \\ Faculty of Sciences, \\ Novi Sad, Serbia
}

\begin{abstract}
:
Cumulative prospect theory (CPT) has provided one of the most influential accounts of how people make decisions under risk. CPT is the model for comparing two decisions amounts regarding two pairs of sets, the positive and negative features of the alternatives. The premium principle is a rule for assigning a premium to an insurance risk. In this article a general CPT premium principle is introduced and characterized. It covers many different types premium principles used in actuarial science, e.g. distortion premium principle, the net premium principle, the asymmetric Choquet integral based premium principle.
\end{abstract}

Keywords:

decision making, general premium principle, Choquet integral, CPT-like integral

\section{INTRODUCTION}

Decision making involves two distinct parties: the decision maker and the decision analyst. Analysts are often tempted to study a problem, and then separately develop an elaborate mathematical model to be used by the decision maker. In decision theory, under the bipolar perspective, Cumulative Prospect Theory (CPT), proposed in [19], is the numerical model for comparing two decisions amounts regarding two pairs of sets, the positive and negative features of the alternatives. In this article a general CPT premium principle is introduced and characterized. It covers many different types premium principles used in actuarial science, e.g. distortion premium principle, the net premium principle, the asymmetric Choquet integral based premium principle. For more details see [13].In the following images the examples of planning two major railway lines in the world are shown. The first relates to the Integration of the Trans Siberian Rail into Euroasian Rai Network (Image 2).

The paper is organized as follows: Section 2. provides different types of decision models and definition of Cumulative prospect theory. Section 3. gives mathematical background. Section 4. introduces the CPT-like integral-based premium principle, provides examples and shows the main properties of the CPT-like integral based premium principle. 


\section{DECISION THEORY CPT}

Decision making is the most important task of a manager and it is often a very difficult one. The foundations of decision making are mainly due to John von Neumann and Oskar Morgenstern.

There are different types of decision models that help to analyze the different scenarios:

- Decision making under uncertainty

- Decision making under risk

- Decision making by buying information (pushing the problem towards the deterministic "pole").

Decisions under uncertainty are relevant in many fields, including finance, economics, psychology, management science, medicine, computer science and engineering. One plausible way to make decisions under uncertainty is by choosing probabilities, sometimes called subjective, for the uncertain events, and then maximizing expected value with respect to those probabilities.

Rank dependence and prospect theory are the best performing and mostly confirmed empirical models

for decisions under risk and uncertainty to date, see [16;20]. In 1992, the psychologists Tversky and Kahneman incorporated Quiggin's idea of rank dependence to solve a theoretical problem of their original prospect theory of 1979. It led to the present version of prospect theory, also called cumulative prospect theory, see [19]. Original prospect theory has some theoretical problems in the way it implemented nonadditive probabilities. It deals only with risk (known probabilities) and within risk it deals only with a limited set of prospects (only two nonzero outcomes), see [16]. The major difference of cumulative prospect theory from its original version is that the concept of weighting is introduced in the cumulative probability distribution function as it is done in the rank-dependent expected utility theory.

In decision theory, under the bipolar perspective there are two weighting functions, $\mathrm{w}+$ for gains and $\mathrm{w}-$ for losses. As for risk, $\mathrm{w}+$ relates to gain- ranked events and $\mathrm{w}-$ relates to loss-ranked events, where we expect $\mathrm{w}+$ and $\mathrm{w}-$ to exhibit similar properties. CPT permits that outcomes are not distinct in the notation $\left(\mathrm{x}_{1}, \mathrm{p}_{1} ; \ldots ; \mathrm{x}_{\mathrm{n}}\right.$, $\mathrm{p}_{\mathrm{n}}$ ) for prospects.
Suppose that $x_{1} \leq \ldots \leq x_{k} \leq 0 \leq x_{k+1} \leq \ldots \leq x_{n}$. Then the CPT value of the prospect $\left(\mathrm{x}_{1}, \mathrm{p}_{1} ; \ldots ; \mathrm{x}_{\mathrm{n}}, \mathrm{p}_{\mathrm{n}}\right)$ is given by the following formula:

$$
\sum_{i=1}^{k} \pi_{i}^{-} \nu\left(x_{i}\right)+\sum_{i=\kappa+1}^{n} \pi_{i}^{+} \nu\left(x_{i}\right)
$$

where the decision weights are defined by:

$$
\begin{aligned}
& \pi_{1}^{-}=w^{-}\left(p_{1}\right) \\
& \pi_{i}^{-}=w^{-}\left(p_{1}+\ldots+p_{i}\right)+w^{-}\left(p_{1}+\ldots+p_{i-1}\right), \\
& 2 \leq i \leq k \\
& \pi_{i}^{+}=w^{+}\left(p_{i}+\ldots+p_{n}\right)-w^{+}\left(p_{i+1}+\ldots+p_{n}\right), \\
& k+1 \leq i \leq n-1
\end{aligned}
$$

Cumulative prospect theory adopts the rankdependent method for transforming probabilities that was introduced in [16], see [20].

\section{MATHEMATICAL BACKGROUND}

Let us consider a class of insurance risks,

$\mathrm{f}: \Omega \rightarrow \mathrm{R}$ (random variables) on a measurable space $(\Omega, \Sigma)$, where $\Sigma$ is a $\sigma$-algebra of subsets of $\Omega, p: \Sigma \rightarrow[0,1]$ is a probability measure, and + denotes the class of all nonnegative insurance risks.

Definition 1. A set function $\mathrm{m}: \Sigma \rightarrow[0, \infty]$ is called a capacity (monotone set function) if $m(\varnothing)=0$ and $m(\mathrm{~A}) \leq m(\mathrm{~B})$, whenever $\mathrm{A} \subseteq \mathrm{B} \subseteq \Omega$ (monotonicity). If in addition $\mu(\Omega)=1$ the capacity is normalized.

We denote by $\boldsymbol{M}$ the set of all $m$ capacities on

$\Omega$.If $m$ does not satisfy monotonicity, it is called a game. For any capacity $m$, the dual capacity is defined by

$$
\bar{m}(A)=m(\Omega)-m(\Omega \backslash A),
$$

for any $\mathrm{A} \subseteq \Omega$.

Let $m$ be a capacity on $\Omega$ and $\mathrm{A}, \mathrm{B} \subseteq \Omega$. We say that $m$ is additive if

$$
m(A \cup B)=m(A)+m(B),
$$

whenever $\mathrm{A}$ and $\mathrm{B}$ are disjoint. 
In the finite case we have the following representation for the Choquet integral:

Let $m$ be a capacity on $\Omega=\{1,2, \ldots, \mathrm{n}\}$ and $\mathrm{x}=\left(\mathrm{x}_{1}, \mathrm{x}_{2}, \ldots\right.$, $\mathrm{x}_{\mathrm{n}}$ ). The Choquet integral of $\mathrm{x} \in[0,1]^{\mathrm{n}}$ with respect to $m$ is defined as:

$$
C_{m}(x)=\sum_{i=1}^{n}\left(x_{\sigma(i)}-x_{\sigma(i-1)}\right) m\left(A_{\sigma(i)}\right),
$$

where $\sigma$ is permutation on $\{1,2, \ldots, \mathrm{n}\}$ such that $\mathrm{x}_{\sigma(1)} \leq \mathrm{x}_{\sigma(2)} \ldots \leq \mathrm{x}_{\sigma(\mathrm{n})}$ with the convention $\mathrm{x}_{\sigma(0)}=0$ and $\mathrm{A}_{\sigma(\mathrm{i})}=\{\sigma(1), \sigma(2), \ldots, \sigma(\mathrm{n})\}$.

According to $[2,3,8,9,14]$, we define the Choquet integral I : $\mathcal{F}$ x $\mathcal{M} \rightarrow[0, \infty]$ of a $\Sigma$-measurable

function $f: \Omega \rightarrow[0, \infty]$ based on a monotone measure $m$.

Definition 2. The Choquet integral I : $\mathcal{F}$ x $\mathcal{M} \rightarrow[0, \infty]$ is defined by:

$$
I(f, m)=\int_{0}^{\infty} m(\{\omega \mid f(\omega) \geq x\}) d x .
$$

The Choquet integral is a special type of the universal integral, introduced in $[8]$, related to the Choquet integral, see $[1 ; 2 ; 3 ; 4 ; 16 ; 17 ; 19 ; 20 ; 21]$. The Choquet integral was introduced in 1953 by Choquet, see [2]. It is a generalization of the Lebesgue integral where the classical measure is replaced by a capacity, see [10].

\section{CPT-LIKE INTEGRAL-BASED PREMIUM PRINCIPLE}

The integral-based premium principle is any rule $\Pi\left(f, \mathrm{~m}_{1}, \mathrm{~m}_{2}\right)$ for assigning a premium $\Pi\left(f, \mathrm{~m}_{1}, \mathrm{~m}_{2}\right)$ to an insurance risk $f: \Omega \rightarrow \mathrm{R}$, and $m_{1}, m_{2} \in \mathcal{M}$. Let $\mathcal{F}_{\mathrm{a}}$ denotes the class of insurance risks $f: \Omega \rightarrow[-\mathrm{a}, \mathrm{a}], \mathrm{a} \in \mathrm{R}^{+}$. For $f \in \mathcal{F}_{\text {a }}$, we denote by $f^{+}, f^{-} \in \mathcal{F}_{\text {a }}^{+}$positive part and the negative part of $f$ defined with $f^{+}=f \vee 0, f^{-}=-f \vee 0$.

Let $\mathrm{Mb}$ denotes class of all monotone measures such that $m(\Omega)=\mathbf{b}$

We introduce the next general CPT type integral:

Definition 3. The CPT-like integral-based premium principle

$\Pi_{\text {CPT }}\left(f, m_{1}, m_{2}\right): \mathcal{F}_{a} \times \boldsymbol{M}_{b} \times \boldsymbol{M}_{b} \rightarrow \mathrm{R}$ for an insurance risk $f \in \mathcal{F}_{\mathrm{a}}$ and $m_{1}, m_{2} \in \boldsymbol{M}_{b}$ is defined by:

$$
\begin{aligned}
& \Pi_{C P T}\left(f, m_{1}, m_{2}\right)= \\
& =\phi^{-1}\left(I\left(\phi\left(f^{+}\right), m_{1}\right)-I\left(\phi\left(f^{-}\right), m\right.\right. \\
& 2
\end{aligned}
$$

for an odd, increasing, continuous function

$$
\phi:[-a, a] \rightarrow[-\infty, \infty], \phi(0)=0, \phi(a)=\infty .
$$

variable at a certain point, but this method can also[13]:

- Calculate the variance of the variable, which enables determination of the estimated size of confidence in intervals;

- Assess an average value of the variable in a section of the area,

- Determine the best location for a new measurement point before planning a network of measuring points.

The software program, which used ArcGIS in the process of the interpolation method, will be offered an opportunity to be cross validated in order to reach the optimal parameters for the selection of reference points. Cross Validation is used for quality assessment or validation of results to predict the applied method of interpolation. It is a process where the interpolation, using the selected parameters (and interpolation methods), interpolates desired value (predicted value) in a reference point, on the condition that the item is not taking part in the interpolation. Different measures and predicted values are to be made afterwards. The procedure is repeated for all locations at which the measurement was performed.

\subsection{Examples}

The CPT-like integral-based premium principle covers many different types premium principles used in actuarial science, see $[5 ; 7 ; 23]$ :

1.The net premium principle, is defined for $f \in \mathcal{F}$ and a probability measure $p \in \mathcal{M}_{1}$ as:

$$
E(f)=\prod_{N}(f, p, p)=I\left(f^{+}, p\right)-I\left(f^{-}, p\right) .
$$

2.The distortion premium principle, is defined for $f \in \mathcal{F}$ and $p \in \mathcal{M}_{1}$ as:

$$
\prod_{D P P}(f, m, m)=I\left(f^{+}, m\right)-I\left(\overline{f^{-}}, m\right),
$$

where $m=g \circ p$ for a non-decreasing distortion function $\mathrm{g}:[0,1] \rightarrow[0,1], \mathrm{g}(0)=0, \mathrm{~g}(1)=1$

3. The asymmetric Choquet integral-based premium principle, is defined for $f \in \mathcal{F}$ and a monotone measure measure $m \in \mathrm{M}_{b}$ as: 


$$
\left.\prod_{A C H}(f, m, m)=I^{+}, m\right)-I\left(f^{-}, \bar{m}\right),
$$

where $\mathrm{m}$ is a dual set function of $\overline{\mathrm{m}}$.

4. The general CPT premium principle, is defined for $f \in \mathcal{F}$ and monotone measures $m_{1}, m_{2} m \in \mathcal{M}_{b}$ as:

$$
\prod_{C P T}\left(f, m_{1}, m\right)=2 I\left(f^{+}, m\right)-I\left(f^{-}, \bar{m}\right) .
$$

Some premium principles do not have the same shape as the above principles, although they have representation in the terms of the monotone integral:

5. The mean premium principle, is defined for $f \in \mathcal{F}$ , a probability measure $p \in \boldsymbol{M}_{1}$ and an increasing real-valued function, $\phi, \phi(0)=0$, as:

$$
\prod_{M}(f)=\phi^{-1}(I(\phi(f), p)) .
$$

6. The exponential premium principle, defined for $f \in \in_{\mathcal{F}}$, a probability measure $\mathrm{p} \in \boldsymbol{M}_{1}$ and $\alpha>0$ by:

$$
\prod_{\text {exp }}(f)={ }_{\bar{\alpha}}^{1} \ln \left(I\left(e^{\alpha f}, p\right)\right) .
$$

The exponential premium principle satisfies many nice properties, including additivity with respect to independent risks. For more details about this integral-based premium principles see $[16 ; 19 ; 20 ; 22]$.

\subsection{Properties of CPT-like integral-based premium} principle

We introduce the following notions:

Let $\phi_{:[-a, a]} \rightarrow[-\infty, \infty]$ be an odd, strictly increasing, continuous function.

The symmetric pseudo-addition $\oplus:[-a, a]^{2} \rightarrow[-a, a]$ is defined by:

$$
x \oplus y=\phi^{-1}(\phi(x)+\phi(y)),
$$

with the convention $\infty-\infty=\infty$

The symmetric pseudo multiplication $\otimes:[-a, a]^{2} \rightarrow[-a, a]$ is defined by:

$$
x \otimes y=\phi^{-1}(\phi(x) \phi(y)),
$$

with the convention $0 \cdot \infty=0$

Let $f$ and $h$ be from $\mathcal{F}_{\mathrm{a}}$. We say that:

$f$ and $h$ are comonotonic risks if

$$
f(w)<f\left(w_{1}\right) \Rightarrow h(w) \leq h\left(w_{1}\right),
$$

for all $w, w_{1} \in \Omega$, see [14;17]. $f$ and $h$ are cosigned risks if $f(w) h(w) \geq 0$, for all $w \in \Omega$.
Let us denote by $f \sim s h$ a couple of comonotonic and cosigned risks. For more details see [11].

The CPT-like integral-based premium principle satisfies the following properties, see [13]:

- Risk loading: for all $m_{1}=m_{2}=p=\boldsymbol{M}_{1}$ and $\phi$ convex on $[0$, a [ we have $\mathrm{E}(f) \leq \Pi\left(f, m_{1}, m_{2}\right)$ for all $f \in \mathcal{F}_{\mathrm{a}}^{+}$.

- Maximal loss: for all $f \in \mathcal{F}_{\mathrm{a}}^{+}$and $m_{1}, m_{2} \in \boldsymbol{M}_{1}$ we have $\Pi\left(f, m_{1}, m_{2}\right) \leq e s s \sup _{m 1}(f)$.

- No unjustified risk loading: if a risk $\mathrm{f}$ is identically equal to a constant $c \in[-\mathrm{a}, \mathrm{a}]$ and $m_{1}, m_{2} \in \boldsymbol{M}_{1}$, than

$\Pi\left(f, m_{1}, m_{2}\right)=c$.

- $\phi$-co-comonotonic additivity: for all $f_{1}, f_{2} \in \mathcal{F}_{\mathrm{a}}$ such that $f_{1} \sim \mathrm{s} f_{2}$ and $m_{1}, m_{2} \in \boldsymbol{M}_{\mathrm{b}}$, it holds

$$
\begin{aligned}
& \Pi\left(f_{1} \oplus f_{2}, m_{1}, m_{2}\right)= \\
& =\Pi\left(f_{1}, m_{1}, m_{2}\right) \oplus \Pi\left(f_{2}, m_{1}, m_{2}\right) .
\end{aligned}
$$

- $\phi$-scale invariance:

$$
\Pi\left(c \otimes f, m_{1}, m_{2}\right)=c \otimes \Pi\left(f, m_{1}, m_{2}\right) .
$$

\section{CONCLUSION}

We have introduced a general premium principle. This new integral-based premium principle can be viewed as a common frame for the mean premium principle and the general CPT premium principle. The future work will be further research into the properties of the general premium principle and its applications.

\section{REFERENCES}

[1] R.J. Aumann and L.S. Shapley. Non-Atomic Games. Princton Univ. Press, Princton, New Jersey, 1974.

[2] G. Choquet. Theory of capacities. Ann. Inst. Fourier 5, 131-295, 1954.

[3] D. Denneberg. Non-additive Measure and Integral. Kluwer Academic Publishers, Dordrecht, 1994.

[4] M. Grabisch, J.L. Marichal, R. Mesiar, and E. Pap. Aggregation Functions. Encyclopedia of Mathematics and its Applications, No 127, Cambridge University Press, 2009. 
[5] T. Grbić, S. Medić, A. Perović, B. Mihailović, N. Novković, and N. Duraković. A Premium Principle Based on the g-integral. Stochastic Analysis and Applications, http://dx.doi.org/10.1080/07362994.2016 .1267574 .

[6] S. Greco, R. Mesiar, and F. Rindone. Discrete bipolar universal integrals. Fuzzy Sets and Systems, 252:55-65, 2014.

[7] M. Kaluszka and M. Krzeszowiec. Pricing insurance contracts under Cumulative Prospect Theory. Insurance: Mathematics and Economics, 50:159-166, 2012.

[8] E.P. Klement, R. Mesiar, and E. Pap. A Universal Integral as Common Frame for Choquet and Sugeno Integral. IEEE Transactions on Fuzzy Systems, 18: 178-187, 2010.

[9] E.P. Klement, J. Li, R. Mesiar, and E. Pap. Integrals based on monotone set functions. Fuzzy Sets and Systems, 281:88-102, 2015.

[10] R. Mesiar, J. Li, and E. Pap. The Choquet integral as Lebesgue integral and related inequalities. Kybernetika, 46:1098-1107, 2010.

[11] B. Mihailović and E. Pap. Asymmetric integral as a limit of generated Choquet integrals based on absolutely monotone real set functions. Fuzzy Sets and Systems, 181:39-49, 2011.

[12] B. Mihailović and P. Đapić. Premium principles based on generated Choquet - integrals. Proceedings of 11th IEEE International Symposium on Intelligent systems and Informatics, pages Subotica, Serbia, 195-198, 2013.

[13] B.Mihailović, E. Pap, M. Štrboja, A. Simićević. A unified approach to the monotone integral-based premium principles under the CPT theory. Stochastic Analysis and Applications, 2017., unpublished.
[14] E. Pap. Null-Additive Set Functions. Kluwer Academic Publishers, Dordrecht, 1995.

[15] E. Pap and M. Štrboja. Generalization of the Jensen inequality for pseudo- ${ }^{\vee}$ integral. Information Sciences, 180:543-548, 2010.

[16] J. Quiggin. Generalized Expected Utility Theory. The Rank-Dependent Model. Kluwer Academic Publishers, Boston, 1993.

[17] D. Schmeidler. Subjective probability and expected utility without additivity. Econometrica, 57:517587, 1989.

[18] M. Štrboja, E. Pap, and B. Mihailović. A monotone convergence rela ${ }^{v}$ ted to pseudo- integral. Proceedings of 13th IEEE International Symposium on Intelligent systems and Informatics, pages Subotica, Serbia, 95-100, 2015.

[19] A. Tversky and D. Kahneman. Advances in prospect theory. Cumulative representation of uncertainty. J. of Risk and Uncertainty, 5:297-323, 1992.

[20] P.P. Wakker. Prospect Theory: For Risk and Ambiguity. Cambridge University Press, New York, 2010.

[21] Z. Wang and G.J. Klir. Generalized Measure Theory. Springer, Boston, 2009.

[22] M.E. Yaari. The dual theory of choice under risk. Econometrica, 55:95-115, 1987.

[23] V.R. Young. Premium principles. Encyclopedia of Acturial Science, John Wiley \& Sons, Ltd, 2006. 